\title{
On the number of partial Steiner systems
}

Armen S. Asratian and N. N. Kuzjurin

The self-archived postprint version of this journal article is available at Linköping University Institutional Repository (DiVA):

http:// urn.kb.se/ resolve?urn=urn:nbn:se:liu:diva-143286

N.B.: When citing this work, cite the original publication.

Asratian, A. S., Kuzjurin, N. N., (2000), On the number of partial Steiner systems, J ournal of combinatorial designs (Print), 8(5), 347-352. https:/ / doi.org/ 10.1002/ 1520-

6610(2000)8:5\&lt;347::AID-J CD4\&gt;3.0.CO;2-8

Original publication available at:

https:/ / doi.org/ 10.1002/ 1520-6610(2000)8:5\&lt;347::AID-J CD4\&gt;3.0.CO;2-8

Copyright: Wiley (12 months)

http:// eu.wiley.com/WileyCDA/ 


\title{
On The Number of Partial Steiner Systems
}

\author{
A. S. Asratian ${ }^{1}$, N. N. Kuzjurin ${ }^{2}$ \\ ${ }^{1}$ Department of Mathematics, Lulea University, S-971 87 Lulea, Sweden, \\ ${ }^{2}$ Institute for System Programming, Russian Academy of Sciences, B. Kommunis- \\ ticheskaya, 25, 109004 Moscow, Russia
}

Received May 25, 1999; accepted November 19, 1999

\begin{abstract}
We give a simple proof of the result of Grable on the asymptotics of the number of partial Steiner systems $\mathbf{S}(\mathbf{t}, \mathbf{k}, \mathbf{m})$. (C) 2000 John Wiley \& Sons, Inc.J Combin Designs $8: 347-352,2000$
\end{abstract}

Keywords: partical Steiner system; matching; hypergraph

\section{INTRODUCTION}

A partial Steiner system $S(t, k, m)$ is a collection of $k$-subsets of an $m$-element set $M$ such that each $t$-subset is contained in at most one $k$-subset from $S(t, k, m)$. When every $t$-subset of $M$ is contained in exactly one $k$-subset from $S(t, k, m)$, we have a classical Steiner system on the set $M$ with parameters $t$ and $k$. Some bounds of the number of such systems for $t=2, k=3$ and $t=3, k=4$ were obtained in [1], [9], [7] and [6]. Very little is known about the number of classical Steiner systems for large $t$ and $k$.

The number of distinct partial Steiner systems $S(t, k, m)$ we denote by $s(t, k, m)$. For two sequences $f_{m}$ and $g_{m}$ we write $f_{m} \sim g_{m}$ if $f_{m} / g_{m} \rightarrow 1$ as $m \rightarrow \infty$.

In [5] Grable announced that using the Rödl nibble algorithm [8] and generalizing the result in [3] he proved the following:

Theorem 1. Let $t$ and $k$ be two fixed positive integers, $t<k$. Then

$$
\ln s(t, k, m) \sim \frac{k-t}{(k)_{t}} m^{t} \ln m \text { as } m \rightarrow \infty
$$

where $(k)_{t}=k(k-1) \ldots(k-t+1)$. 
The Rödl nibble algorithm is a very powerful but not an easy technique. In this paper we give a simple proof of Theorem 1. We use the result of Frankl and Rödl [4] concerning the existence of nearly perfect matchings in hypergraphs. A hypergraph $H$ is a pair $(V, E)$, where $V$ is a finite set of vertices and $E$ is a finite family of subsets of $V$, called edges. A hypergraph is $r$-uniform if every edge contains precisely $r$ vertices. The number of edges of a hypergraph $H$ containing a vertex $v$ is called the degree of $v$ and denoted by $d_{H}(v)$ or simply $d(v)$. For two distinct vertices $u$ and $v$ of a hypergraph $H$, the number of edges containing both $u$ and $v$ is denoted by $d_{H}(u, v)$ or simply $d(u, v)$. A matching in a hypergraph is a collection of pairwise disjoint edges. We will use the result in [4] in the following slightly weaker form.

Theorem 2. Let integer $r \geq 3$ and real $\delta>0$ be fixed, and $H$ be an r-uniform hypergraph on $n$ vertices. There exists $\gamma>0$ and $n_{0}>0$ such that if for some $D$ and for every pair of distinct vertices $u$ and $v$ of $H$ the following two conditions hold:

(1) $(1-\gamma) D \leq d(v) \leq(1+\gamma) D$,

(2) $d(u, v) \leq \bar{D} /(\ln n)^{4}$,

then for all $n \geq n_{0} H$ has a matching containing at least $\left\lceil(1-\delta) \frac{n}{r}\right\rceil$ edges.

\section{PROOF OF THEOREM 1}

Let $r=\left(\begin{array}{l}k \\ t\end{array}\right), n=\left(\begin{array}{c}m \\ t\end{array}\right), d=\left(\begin{array}{c}m-t \\ k-t\end{array}\right)$, and $q=\left(\begin{array}{c}m \\ k\end{array}\right)$. Consider the family $F=\left\{A_{1}, \ldots, A_{q}\right\}$ of all $k$-subsets of an $m$-set $M$. We define the hypergraph $H(t, k, m)$ corresponding to the family $F$ in the following way: The vertex set $V$ of $H(t, k, m)$ is the set of all $t$-subsets of $M$ and the edge set is $E=\left\{e_{1}, \ldots, e_{q}\right\}$, where each $e_{i}$ consists of all $t$-subsets which are contained in a $k$-subset $A_{i}$. For each matching $\left\{e_{i_{1}}, \ldots, e_{i_{l}}\right\}$ of $H(t, k, m)$ the corresponding set $\left\{A_{i_{1}}, \ldots, A_{i_{l}}\right\}$ in $F$ is a partial Steiner system $S(t, k, m)$. Since this correspondence is one-to-one, the number $s(t, k, m)$ of all partial Steiner systems is equal to the number of matchings in the hypergraph $H(t, k, m)$. Note, that each edge of the hypergraph $H(t, k, m)$ contains exactly $r=\left(\begin{array}{l}k \\ t\end{array}\right)$ vertices, that is, the hypergraph is $r$-uniform.

Let $\delta$ be real, $0<\delta<1 / 2$. We define a random subfamily $F(p)$ of the family $F$ by choosing independently each $k$-subset of $F$ with probability $p=d^{-1+\delta}$. Taking into account the one-to-one correspondence between $k$-subsets of the $m$-set $M$ and the edges of the hypergraph $H(t, k, m)$ we obtain a random subhypergraph $H_{p}=H_{p}(t, k, m)$ corresponding to the random family $F(p)$.

Lemma 1. Let $X$ be the random variable equal to the number of partial Steiner systems $S(t, k, m)$ in the random family $F(p)$ each containing at least $T=\left\lceil(1-\delta) \frac{n}{r}\right\rceil$ $k$-subsets. Then, $\mathbf{P}\{X \geq 1\} \geq 1-\delta$ for sufficiently large $m$.

The proof of Lemma 1 will be given in Section 3. It uses the observation that since the vertex degrees and pair degrees of random subhypergraph $H_{p}$ are sums of independent indicator variables, the Chernoff bounds prove that $H_{p}$ almost always satisfy the conditions of Theorem 2 and, therefore, contains a matching with at least $T=\left\lceil(1-\delta) \frac{n}{r}\right\rceil$ edges. 
Let $N$ be the number of partial Steiner systems $S(t, k, m)$ each containing at least $T$ $k$-subsets. Let us compare upper and lower bounds of the probability $\mathbf{P}\{X \geq 1\}$.

A lower bound. By Lemma 1

$$
\mathbf{P}\{X \geq 1\} \geq 1-\delta
$$

Markov's inequality shows that

$$
\mathbf{P}\{X \geq 1\} \leq \mathbf{E} X \leq N p^{T}
$$

Thus,

$$
N \geq(1-\delta) p^{-T}
$$

which implies the inequality

$$
s(t, k, m) \geq N \geq(1-\delta) d^{(1-\delta)^{2 n / r}} .
$$

An upper bound. The number of matchings $s(t, k, m)$ satisfies the following trivial upper bound:

$$
s(t, k, m) \leq \sum_{j=1}^{\left\lceil\frac{n}{r}\right\rceil}\left(\begin{array}{l}
q \\
j
\end{array}\right) \leq \frac{n}{r}\left(\begin{array}{c}
q \\
\left\lceil\frac{n}{r}\right\rceil
\end{array}\right) .
$$

Taking into account that $\left(\begin{array}{l}q \\ j\end{array}\right) \leq\left(\frac{e q}{j}\right)^{j}$, and $q=\frac{n d}{r}$ we obtain

$$
s(t, k, m) \leq \frac{n}{r}\left(\begin{array}{c}
\frac{n d}{r} \\
\left\lceil\frac{n}{r}\right\rceil
\end{array}\right) \leq \frac{n}{r}(e d)^{n / r} \leq d^{(1+\delta) n / r} .
$$

Combining the inequalities (1) and (2) which hold for any fixed $0<\delta<\frac{1}{2}$ and sufficiently large $m$, we obtain that

$$
\ln s(t, k, m) \sim \frac{n}{r} \ln d \text { as } m \rightarrow \infty .
$$

Taking into account that

$$
\frac{n}{r}=\frac{(m)_{t}}{(k)_{t}}, \quad(m)_{t} \sim m^{t}, \quad \ln d=\ln \left(\begin{array}{c}
m-t \\
k-t
\end{array}\right) \sim(k-t) \ln m,
$$

we obtain that

$$
\ln s(t, k, m) \sim \frac{k-t}{(k)_{t}} m^{t} \ln m \text { as } \mathrm{m} \rightarrow \infty
$$




\section{PROOF OF LEMMA 1}

Let $D(u)$ be the random variable equal to the degree of vertex $u$ in $H_{p}(t, k, m)$, and for each pair of distinct vertices $u$ and $v$ let $D(u, v)$ be the random variable equal to the number of edges in $H_{p}(t, k, m)$ containing both $u$ and $v$. For all vertices $u, v \in V$ let $A(u)$ denote the event: $|D(u)-\mathbf{E} D(u)|>(\ln d)^{-1} \mathbf{E} D(u)$ and $B(u, v)$ denote the event: $D(u, v)-\mathbf{E} D(u, v)>(\ln n)^{-5} d^{\delta}$. We need the following technical lemma.

Lemma 2. For sufficiently large $m$

$$
\mathbf{P}\{A(u)\} \leq \exp \left\{-d^{\delta / 2}\right\}, \quad \mathbf{P}\{B(u, v)\} \leq \exp \left\{-d^{\delta / 2}\right\} .
$$

Proof of Lemma 2. We use the following bounds for probabilities of large deviations of sums $Z=\sum_{i=1}^{L} z_{i}$ of independent random variables $z_{1}, \ldots, z_{L}$ such that $z_{i}$ takes two values 0 and 1 , and $\mathbf{P}\left\{z_{i}=1\right\}=p, \quad \mathbf{P}\left\{z_{i}=0\right\}=1-p$ (see [2]):

$$
\begin{gathered}
\mathbf{P}\{|Z-\mathbf{E} Z|>\varepsilon \mathbf{E} Z\} \leq 2 \exp \left\{-\left(\varepsilon^{2} / 3\right) \mathbf{E} Z\right\}, \quad \text { if } 0 \leq \varepsilon \leq 1, \\
\mathbf{P}\{Z-\mathbf{E} Z>\varepsilon \mathbf{E} Z\} \leq \exp \{(\varepsilon-(1+\varepsilon) \ln (1+\varepsilon)) \mathbf{E} Z\}, \quad \text { if } \varepsilon \geq 0 .
\end{gathered}
$$

For each vertex $u$ we define independent random variables $y_{1}, \ldots, y_{d(u)}$ such that $y_{j}=1$ iff the $j$ th edge containing $u$ is in $H_{p}(t, k, m)$, and $y_{j}=0$, otherwise. It is clear, that

$$
D(u)=\sum_{j=1}^{d(u)} y_{j}, \quad \mathbf{E} D(u)=\sum_{j=1}^{d(u)} \mathbf{E} y_{j}=p d=d^{\delta}
$$

Using (3) we obtain

$$
\mathbf{P}\left\{|D(u)-\mathbf{E} D(u)|>(\ln d)^{-1} \mathbf{E} D(u)\right\} \leq 2 \exp \left\{-(2 \ln d)^{-2} d^{\delta}\right\} \leq \exp \left\{-d^{\delta / 2}\right\} .
$$

Similarly, for every two distinct vertices $u$ and $v$ we define independent random variables $x_{1}, \ldots, x_{d(u, v)}$ such that $x_{j}=1$ iff the $j$ th edge containing both $u$ and $v$ is in $H_{p}(t, k, m)$, and $x_{j}=0$ otherwise. Clearly,

$$
D(u, v)=\sum_{j=1}^{d(u, v)} x_{j}
$$

Since $d=\left(\begin{array}{c}m-t \\ k-t\end{array}\right) \leq(m-t)^{k-t}$, we obtain, for sufficiently large $m$,

$$
\mathbf{E} D(u, v) \leq p \max _{u \neq v} d(u, v) \leq p\left(\begin{array}{c}
m-t-1 \\
k-t-1
\end{array}\right) \leq(k-t) d^{\delta-1 /(k-t)} \leq d^{\delta}(\ln n)^{-6} .
$$


Let $d^{\delta}=g \mathbf{E} D(u, v), \theta=(\ln n)^{-5}$. Then (5) implies $\theta g>\ln n$. This and (4) imply

$$
\begin{aligned}
& \mathbf{P}\left\{D(u, v)-\mathbf{E} D(u, v)>\theta d^{\delta}\right\}=\mathbf{P}\{D(u, v)-\mathbf{E} D(u, v)>\theta g \mathbf{E} D(u, v)\} \\
& \quad \leq \exp \{(\theta g-(1+\theta g) \ln (1+\theta g)) \mathbf{E} D(u, v)\} \\
& \quad \leq \exp \{-\theta g(\ln (\theta g)-1) \mathbf{E} D(u, v)\} \leq \exp \left\{-d^{\delta}(\ln n)^{-5}\right\} \leq \exp \left\{-d^{\delta / 2}\right\} .
\end{aligned}
$$

The proof of Lemma 2 is complete.

Lemma 2 and inequality (4) imply, for sufficiently large $m$,

$$
\begin{gathered}
\mathbf{P}\left\{\bigcup_{u \in V} A(u)\right\} \leq n \max _{u} \mathbf{P}\{A(u)\} \leq m^{t} \exp \left\{-m^{\delta / 4}\right\} \leq \delta / 2, \\
\mathbf{P}\left\{\bigcup_{u, v \in V, u \neq v} B(u, v)\right\} \leq n^{2} \max _{u \neq v} \mathbf{P}\{B(u, v)\} \leq m^{2 t} \exp \left\{-m^{\delta / 4}\right\} \leq \delta / 2 .
\end{gathered}
$$

Note, that the event $\overline{\bigcup_{u \in V} A(u)}$ implies, for every vertex $u$, the inequality

$$
\left|D(u)-d^{\delta}\right| \leq(\ln d)^{-1} d^{\delta}
$$

and the event $\overline{\bigcup_{u, v \in V, u \neq v} B(u, v)}$ implies for every two distinct verticies $u, v$ the inequality

$$
D(u, v) \leq \mathbf{E} D(u, v)+(\ln n)^{-5} d^{\delta} \leq 2 d^{\delta}(\ln n)^{-5} .
$$

Let $\gamma$ and $n_{0}$ be the constants in Theorem 2. It is clear that for sufficiently large $m$ the inequality (6) implies the condition 1 of Theorem 2 with $D=d^{\delta}$, and the inequality (7) implies the condition 2 of Theorem 2 with $D=d^{\delta}$. Thus, by Theorem 2

$$
\mathbf{P}\{X \geq 1\} \geq 1-\left(\mathbf{P}\left\{\bigcup_{u \in V} A(u)\right\}+\mathbf{P}\left\{\bigcup_{u, v \in V, u \neq v} B(u, v)\right\}\right) \geq 1-\delta .
$$

\section{ACKNOWLEDGMENTS}

We thank Svenska Institute for the financial support. We also thank the referees for their valuable remarks.

\section{REFERENCES}

[1] V. E. Aleksejev, On the number of Steiner triple systems, Math. Notes 15 (1974), 461-464.

[2] N. Alon and J. H. Spencer, The Probabilistic Method, Wiley, 1992.

[3] C. J. Colbourn, D. G. Hoffman, K. T. Phelps, V. Rödl, and P. M. Winkler, The number of twise balanced designs, Combinatorica 11 (3) (1991), 207-218.

[4] P. Frankl and V. Rödl, Near perfect coverings in graphs and hypergraphs, European J. 9 Combinatorics 6 (1985), 317-326. 
[5] D. A. Grable and K. T. Phelps, Random methods in design theory: a survey, J. Comb. Designs 4 (4) (1996), 255-273.

[6] H. Lenz, On the number of Steiner quadruple systems, Mitt. Math. Sem. Giessen 169 (1985), 55-71.

[7] K. T. Phelps, On the number of commutative Latin squares, ARS Combinatoria 10 (1980), 311-322.

[8] V. Rödl, On a packing and covering problem, European J. Combinatorics 5 (1985), 69-78.

[9] R. M. Wilson, Nonisomorphic Steiner triple systems, Math. Z. 135 (1974), 303-313. 